
\title{
$\checkmark$ Research Square \\ The BAG2 and BAG6 Genes are Involved in Multiple Abiotic Stress Tolerance in Arabidopsis Thaliana
}

\author{
Muhammad Arif \\ Nankai University College of Life Sciences \\ Zitong Li \\ Nankai University College of Life Sciences \\ Qiong Luo \\ Nankai University College of Life Sciences \\ Luhua Li \\ Nankai University College of Life Sciences \\ Yuequan Shen \\ Nankai University College of Life Sciences \\ Shuzhen Men ( $\nabla$ shuzhenmen@nankai.edu.cn ) \\ Nankai University https://orcid.org/0000-0001-6472-6829
}

\section{Research article}

Keywords: Arabidopsis thaliana, BAG, drought stress, heat stress, abscisic acid (ABA), reactive oxygen species.

Posted Date: October 7th, 2020

DOI: https://doi.org/10.21203/rs.3.rs-76072/v1

License: (c) (1) This work is licensed under a Creative Commons Attribution 4.0 International License.

Read Full License 


\section{Abstract}

Background: The BAG (Bcl-2 associated athanogene) family is a multi-functional group of proteins that perform functions ranges from apoptosis to environmental stress tolerance. The BAG proteins are found in yeast, plants and animals. In plants, especially in Arabidopsis thaliana (Arabidopsis), BAG proteins were found to play roles both in abiotic and biotic stresses tolerance including heat, cold, salt, drought, and pathogens. However, the function of Arabidopsis BAG2 remains largely unknown. Whereas BAG6 was found to play important roles in plants response to biotic stresses, it remains unknown whether BAG6 is involved in plants tolerance to abiotic stresses.

Results: In this study, we have characterized functions of the Arabidopsis BAG2 and BAG6 genes. Promoter:GUS analysis results show that both $B A G 2$ and $B A G 6$ genes are expressed in various tissues in Arabidopsis thaliana. Expression of both $B A G 2$ and $B A G 6$ genes is induced by salt, mannitol, and heat stress treatments and by the stress-related hormones including ABA, ethylene, and SA, while BAG6 expression is additionally induced by JA. Germination of the bag 2 and bag6 single and bag2 bag6 double mutant seeds are less sensitive to ABA than that of the wild type (WT). Both bag2 and bag6 single mutant and bag 2 bag 6 double mutant show more survival rate than WT in drought treatment but display less survival rate on $45^{\circ} \mathrm{C}$ heat stress experiment. Consistently, transcription levels of stress-related genes such as RD29A, RD29B and NCED3 are higher in the mutant than that in the WT. Furthermore, these mutants exhibit lower content of reactive oxygen species (ROS) after drought and ABA treatment but higher ROS accumulation after heat treatment than the WT.

Conclusion: These results suggest that $B A G 2$ and $B A G 6$ genes are negatively involved in drought stress but play a positive role in heat stress in Arabidopsis.

\section{Background}

The BAG (Bcl-2 associated athanogene) family is a group of multi-functional chaperone proteins that contain a conserved BAG domain at their $\mathrm{C}$-terminal $[1,2,3]$. The BAG proteins are evolutionarily conserved with homologs present in yeast, plants and animals. They perform functions ranges from apoptosis to response to biotic and abiotic stresses. Seven $B A G$ genes were identified in the model plant Arabidopsis thaliana (Arabidopsis) ranges from $B A G 1$ to $B A G 7[4,5]$. The AtBAG1-4 proteins contain a BAG domain and an Ubiquitin-like domain (UBL), whereas the AtBAG5-7 proteins contain an IQ motif, which preferentially binds to $\mathrm{Ca}^{2+}$-free calmodulin (CaM) in addition to a BAG domain [5,6,7]. Phylogenetic analysis by MEGA7 software clearly revealed that the plant BAG family proteins can be divided into two subgroups, Group I including the BAG1-4 proteins, and Group II including the BAG5-7 proteins (Fig. S1). Overall, plant BAG proteins have roles in programmed cell death (PCD), in plants response to biotic and abiotic stresses and in development $[1,3]$.

AtBAG1 was found to be localized in the cytosol and interact with the chaperone Hsc70-4 to cooperatively mediate proteasomal degradation of misfolded cytosolic proteins and unimported plastid proteins [8]. 
Physiologically, AtBAG1 overexpression transgenic seedlings were more sensitive to salt treatment, suggesting that $A t B A G 1$ might be involved in plants response to abiotic stress [8]. It has been reported that under normal growth conditions the atbag2 mutant exhibited slightly better growth than the WT, implying that AtBAG2 might participate in plants PCD or response to environmental stresses [6]. AtBAG3 expression was upregulated by salt stress and by the stress-related hormones ABA and ACC, but downregulated by cold stress [1]. AtBAG3 protein is localized to the cytosol [8]. However, so far the physiological function of $A t B A G 2$ and $A t B A G 3$ remains unknown.

Among the plant $B A G$ genes, $B A G 4$ is one of the most studied. Overexpression of the AtBAG4 in tobacco plants confers tolerance to UVB irradiation and cold, oxidant and salt treatments [5]. In contrast, the atbag 4 mutant is more sensitive to salt treatment [2]. In rice, OsBAG4 functions as a positive regulator of disease resistance, and an E3 ligase EBR1 targets OsBAG4 for proteasome degradation to balance growth and immune response [9]. Recently, AtBAG4 was found to interact with the potassium channel protein KAT1 and help its localization to the plasma membrane [10].

Our previous studies show that AtBAG5 acts as a hub linking calcium signaling and the Hsc70 chaperone to regulate leaf senescence $[7,11]$. AtBAG6 expression is induced by the stress-related plant hormone SA and $A B A$, and by heat stress and atbag 6 mutant is sensitive to pathogen infection and non-acclimated heat stress $[5,1,13]$. However, another report found that atbag6 mutant exhibited mild thermotolerance to non-acclimated heat stress compared to the WT plants [12]. Although in acclimated heat stress treatment, atbag6 mutant showed no different phenotype from those of the WT, it could increase the thermotolerance of the fes1a mutant [13].Overexpression of AtBAG6 induced cell death [14]. Aspartyl protease-mediated cleavage of AtBAG6 is necessary for autophagy and fungal resistance in plants [15]. AtBAG7 localized in ER and is involved in unfolded protein response and heat tolerance $[16,17]$. It has been reported that salt treatment downregulated expression of AtBAG6 and AtBAG7, but ACC treatment could reverse the effect of salinity [18]. Whereas $B A G 6$ was found to play significant role in biotic (pathogens) and abiotic stresses (heat), it remains unknown whether BAG6 is involved in other stressors or not.

Here, we have focused on function of BAG2 and BAG6 in Arabidopsis to analyze their expression pattern in response to various hormones and stresses and especially abiotic stress treatments such as drought, $A B A$ and heat stress and the genes involved in it. Our results showed that both BAG2 and BAG6 genes are expressed in various tissues in Arabidopsis. Expression of both BAG2 and BAG6 genes is induced by salt, mannitol, and heat treatments and by the stress-related hormones including $A B A$, ethylene and $S A$, while $B A G 6$ is additionally induced by JA. Germination of the bag2 and bag6 single and bag 2 bag 6 double mutant seeds are less sensitive to ABA than that of the wild type (WT). Both bag 2 and bag 6 single mutant and bag2 bag 6 double mutant showed more survival rate than WT in drought treatment but displayed less survival rate on $45^{\circ} \mathrm{C}$ heat stress experiment. Consistently, these mutants displayed higher transcription levels of multiple stress-related genes such as RD29A, RD29B and ABA biosynthesis genes NCED3 than that of the WT in response to ABA treatment. Finally, when compared to wild type plants, the 
mutants exhibited higher content of reactive oxygen species (ROS) after heat stress and less contents accumulated after drought and ABA treatment.

\section{Results}

\section{$B A G 2$ and $B A G 6$ expression during Arabidopsis development}

To determine expression patterns of the $B A G 2$ and $B A G 6$ genes, we generated Arabidopsis transgenic lines expressing the GUS reporter gene under control of the promoter fragments of the BAG2 and BAG6 genes, respectively (ProBAG2:GUS and ProBAG6:GUS) and analyzed the expression of the GUS reporter gene in various tissues. Strong GUS staining was observed in most tissues throughout the ProBAG2:GUS and ProBA G6:GUS transgenic plants (Fig. 1 A-T). In 7-day-old seedlings, ProBAG2:GUS activity is strongly detected in cotyledons, hypocotyl, and root vascular tissues, with the strongest signal at the lower part of the hypocotyl (Fig. 1A and B), while ProBAG6:GUS expression is strongly detected in all of the tissues of the whole seedling (Fig. $1 \mathrm{~K}$ and L). In 2-week-old plants and bolting plants, both ProBAG2:GUS and ProBAG6:GUS are highly expressed in rosette leaves and cauline leaves (Fig. 1 C-E and M-O), with ProBAG6:GUS showing additional GUS activity in stems (Fig. 1P). In flowers, both ProBAG2:GUS and ProBAG6:GUS are expressed in sepals, anther filaments, pollen grains, and style (Fig. $1 \mathrm{~F}-\mathrm{H}$ and Q-R), with ProBAG6:GUS showing additional expression in ovules (Fig. 1R). In siliques, both ProBAG2:GUS and ProBAG6:GUS are expressed in young siliques with ProBAG6:GUS showing stronger GUS signals (Fig. 11 and $S$ ), while in old siliques both genes expression are very weak (Fig. $1 \mathrm{~J}$ and $\mathrm{T}$ ).

\section{$B A G 2$ and BAG6 expression response to abiotic stress and plant hormones}

We further investigated whether $B A G 2$ and $B A G 6$ expression is regulated by abiotic stressors or plant hormones. The results show that ProBAG2:GUS expression is strongly increased by mannitol, salt ( $\mathrm{NaCl})$, heat and $A B A$ treatments (Fig. 2A-B; Fig. S2 A) and its expression is also slightly increased in response to other stressors and hormones such as PEG, SA and ACC, respectively. Similarly, ProBAG6:GUS showed significant enhanced GUS staining in response to JA, ABA, mannitol, salt, and heat treatment, while showed slight increase in response to ACC, PEG and SA (Fig. 2C-D; Fig. S2 B-D). These results are similar to a previous report that had analyzed $A t B A G$ genes response to abiotic stressors such as cold, heat, mannitol, and salt and hormones such as ABA, ACC, MeJA and SA by reverse transcription quantitative PCR (RT-qPCR) [1]. Together, these results suggest that AtBAG2 and AtBAG6 genes are involved in plants response to environmental stress. 


\section{Loss-of-function of BAG2 and BAG6 in Arabidopsis improves tolerance to $A B A$ and drought Stresses}

To explore the function of these genes, transfer DNA (T-DNA) insertion mutants were isolated for BAG2 and BAG6 within the fourth exon (SALK_030285, bag2; Fig. 3A) and within the first exon (SALK_004760, bag6; Fig. 3C), respectively. These mutants were crossed to generate the bag 2 bag 6 double mutant. The bag2 and bag6 mutants had been reported previously [6,12]. RT-PCR analyses revealed that weak BAG2 transcript was detected in the homozygous bag2 mutant (Fig. 3B; Fig. S3A-B) and no BAG6 transcript was detectable in the homozygous bag6 mutant (Fig. 3D; Fig. S3C-D), indicating that the bag2 mutant is a knockdown allele and the bag6 mutant is a null allele. Under normal growth conditions, the bag 2 and bag6 single and bag2 bag6 double mutants exhibited slightly larger rosette size than the WT (Fig. S4).

To study the response of bag 2 and bag 6 single and bag 2 bag 6 double mutants to the plant stress hormone abscisic acid (ABA), these mutants and wild type (WT) seeds were sown on Murashige and Skoog (MS) medium supplemented with different concentrations of ABA to compare the germination and greening rate between WT and mutants lines. Without ABA, the WT and mutants showed similar germination rate and greening rate, but when ABA was applied, higher germination and greening rate were observed in the bag2 and bag6 single and bag2 bag 6 double mutants (Fig. 4). When compared germination and greening rate on $0.75 \mu \mathrm{M} \mathrm{ABA}$, we found a significant difference i-e WT germination rate (72\%) and greening rate (46\%) while bag $275 \%$ and $84 \%$, bag $679 \%$ and $88 \%$ and bag 2 bag $681 \%$ and $91 \%$, respectively (Fig. 4B-C). Similarly, when compared on $1 \mu \mathrm{M}$ ABA, WT exhibited $57 \%$ seeds germinated and after 7 days $29 \%$ greening rate were found but bag 2 single mutant exhibited $65 \%$ germination while $49 \%$ greening rate were observed, bag 6 also showed more germination and greening rate than WT (i-e 68\% and 55\%, respectively) (Fig. 4B-C). Interestingly bag2 bag6 double mutant germination rate was more than each single mutant and WT which is $75 \%$ and $71 \%$, respectively (Fig. 4BC). Finally, when compared on $2 \mu \mathrm{M}$ ABA, WT exhibited $37 \%$ germination and $24 \%$ greening rate, while bag2 exhibited $50 \%$ and $36 \%$, bag $65 \%$ and $36 \%$ and bag 2 bag6 $62 \%$ and $49 \%$, respectively (Fig. 4B-C). Thus these mutants appeared to enhance the ABA tolerance of Arabidopsis during germination and the early vegetative growth period.

To study drought stress tolerance, 21-day-old WT and mutants plants were used, and 9 plants per pot were sown and 12 pots were prepared for each genotype (108 plants in total for each genotype). After withholding water for 14 days, almost all the WT and mutants plants were wilted and near to die (Fig. $5 A)$. Then the plants were re-watered for three days. On third day a significant difference were noticed between WT and mutants plants (Fig. 5A); bag2 showed 43\%, bag6 showed 44\%, bag2 bag 6 showed highest survival rate i-e 73\% while WT showed less survival rate i-e $29 \%$ (Fig. 5B). To further verify these results, we performed water loss experiment with different time interval. Consistently, the water loss in mutants plants rosette leaves was slower than that of the WT (Fig. 5C). These results suggest that BAG2 and BAG6 play a negative role in Arabidopsis response to ABA and drought treatments. 


\section{Loss-of-function of BAG2 and BAG6 in Arabidopsis compromises tolerance to heat stress}

Our ProBAG2:GUS and ProBAG6:GUS expression results and previously reported RT-qPCR results showed that $B A G 2$ and $B A G 6$ genes were upregulated during heat stress $[1,13]$. So here in this study we have studied the effect of heat stress on bag 2 and bag 6 single and bag 2 bag 6 double mutants. The results showed that the bag2 and bag6 single and bag 2 bag 6 double mutants are sensitive to heat stress when compared to WT seedlings (Fig. 6). The survival rates of these mutants were one third less than WT (Figs. 6B). Also we measured chlorophyll content in mutants and WT on control untreated seedlings $\left(22^{\circ} \mathrm{C}\right)$ and $45^{\circ} \mathrm{C}$ heat treated seedlings. Significant difference was observed (Figs. 6C). Total chlorophyll levels decreased as consequence of the heat treatment both in WT and mutants, but the mutants retained less chlorophyll contents (Fig. 6C). These results suggest that BAG2 and BAG6 play a positive role in Arabidopsis response to heat stress.

\section{Stress related genes are upregulated in the bag2 and bag6 mutants}

To further investigate the role of $B A G 2$ and $B A G 6$ in plants response to $A B A$, heat and drought treatment, we performed RT-qPCR analysis on several stress-related genes such as $R D 29 A, R D 29 B$ (stress related), and NCED3 (ABA biosynthesis genes). We found that the expression of RD29A and RD29B genes was upregulated in bag 2 and bag 6 single mutants and bag 2 bag 6 double mutant compared to WT when treated with ABA (Fig. 7A-B), while expression of the ABA biosynthesis gene NCED3 was slightly upregulated in these mutants compared to WT (Fig. 7C). These results further support the heat, ABA and drought phenotypic data.

\section{ROS accumulations in WT and mutant plants}

The accumulation of $\mathrm{H}_{2} \mathrm{O}_{2}$ and superoxide anion $\left(\mathrm{O}_{2}^{-}\right)$was detected using 3,3-diaminobenzidine (DAB) and nitro blue tetrazolium (NBT) staining in seedling shoots and roots. Upon $20 \mu \mathrm{M}$ ABA treatment, the cotyledon and root of the mutant plants displayed lighter color than that of the WT, which clearly indicates lower accumulation of $\mathrm{O}_{2}{ }^{-}$and $\mathrm{H}_{2} \mathrm{O}_{2}$, respectively in the mutant seedlings (Fig. 8A-B), whereas upon heat stress treatment, the mutants exhibited higher accumulation of $\mathrm{O}_{2}{ }^{-}$than the WT (Fig. $8 \mathrm{C}$ ). Finally DAB staining was also applied to drought stressed rosette leaves of WT and mutant plants. We got the same results that leaves of mutant lines accumulated lower $\mathrm{H}_{2} \mathrm{O}_{2}$ than that of the WT (Fig. S5). Combined these results indicate that mutant lines had a lower accumulation of ROS when treated with $A B A$ and drought while higher accumulation when treated with heat stress. These results corresponded well with the respective drought- and heat-tolerance phenotypes of these mutants. 


\section{Discussion}

In plants especially in Arabidopsis BAG proteins are a topic of interest and they mediate multiple abiotic, biotic and developmental processes. Actually, most research on plant BAGs so far are focused on their functions in abiotic and biotic stress tolerance. The expression patterns of AtBAG genes and their roles during plant development remain largely unknown. In this study, we analyzed the expression patterns of AtBAG2 and AtBAG6 genes during development by using ProBAG2:GUS and ProBAG6:GUS transgenic lines. Interestingly, the results show that both $A t B A G 2$ and $A t B A G 6$ genes are expressed in various tissues with overlapping or specific expression patterns during the whole developmental process. For example, ProBAG6:GUS is expressed in all of the root tissues with stronger expression in columella root cap cells and root vascular tissue, but ProBAG2:GUS is only expressed in the root vascular tissue. In shoots, both ProBAG2:GUS and ProBAG6:GUS are strongly expressed in rosette leaves and cauline leaves, but they again show differences in flower tissues. Mutant analysis showed that bag2 and bag6 single mutants and bag 2 bag 6 double mutant plants are a bit larger than the WT, and they show no developmental defect. These results suggest that the AtBAG2 and AtBAG6 genes and other AtBAG genes may have redundant and specific functions during Arabidopsis development. Analyzing the other AtBAG genes expression patterns and generating multiple atbag mutants by crossing or by using CRISPR/Cas 9 gene editing methods will provide more information of the role of the AtBAG family during Arabidopsis development.

Previous RT-qPCR and Northern blot analysis had shown that AtBAG2 transcript level was significantly upregulated by $A B A$ and salt treatment and $A t B A G 6$ transcript level was significantly upregulated by $A B A$, $\mathrm{SA}, \mathrm{H}_{2} \mathrm{O}_{2}$ and heat treatment [1,5,13]. Consistently, our ProBAG2:GUS and ProBAG6:GUS expression results show that they both are upregulated by heat, salt and osmotic treatment and by stress-related plant hormones including ABA, SA and ACC treatment, and ProBAG6:GUS is additionally induced by JA. These expression results suggest that the AtBAG2 and AtBAG6 genes may be involved in Arabidopsis response to multiple environmental stresses. Indeed our stress and hormone treatments results show that bag 2 and bag 6 single mutants and bag 2 bag 6 double mutant seeds germination are less sensitive to $A B A$ treatment and their plants are more tolerant to drought stress than the WT, but they are more sensitive to heat stress. Interestingly, in ABA and drought treatments the bag 2 bag 6 double mutant shows more tolerance than either of the single mutant, suggesting an additive effect of these two genes in Arabidopsis response to ABA or drought stress treatments.

\section{Conclusion}

Results in the present study show that the $A t B A G 2$ and $A t B A G 6$ genes are broadly expressed in various tissues during Arabidopsis development. Also, AtBAG2 and AtBAG6 expression is induced by abiotic stresses such as heat, salt and osmotic and by stress-related plant hormones including ABA, SA, JA and ethylene. Seed germination of bag2 and bag 6 single and bag 2 bag 6 double mutant are more tolerant to ABA treatment and their plants are more tolerant to drought stress but sensitive to heat stress. These 
findings suggest that $A t B A G 2$ and $A t B A G 6$ play important roles in Arabidopsis response to multiple abiotic stresses and hormones.

\section{Methods}

\section{Plant Materials and Growth Conditions}

The T-DNA insertion mutants bag2 (SALK_030285) and bag6 (SALK_004760) are in Columbia-0 (Col-0) background. These mutants were obtained from the Arabidopsis Stock Centre and double mutant is obtained by crossing bag2 and bag6. T-DNA insertion was confirmed by RT-PCR analysis. Primers used in this experiment are listed in Supplemental Table S1. Seeds were surface sterilized in $70 \%$ ethanol for $5 \mathrm{~min}$ and $1 \%$ Clorox bleach for $10 \mathrm{~min}$, stratified for 3 days at $4{ }^{\circ} \mathrm{C}$. The seeds were plated on solidified Murashige and Skoog (MS) medium and vertically grown at $22^{\circ} \mathrm{C}$ under $16 \mathrm{~h}$ light/ $8 \mathrm{~h}$ dark conditions. For ABA treatments experiment, all the single, double mutant and WT seeds were surface sterilized and after 72 hours stratification, sown on plates supplemented with or without ABA. For drought treatments, the stratified seeds were grown vertically on MS plates for seven days and transferred to soil $(75 \%$ soil and $25 \%$ vermiculite) for more 14 days to grow. Nine plants per pots were grown. During each independent experiment, we grew 108 plants in total for each genotype.

\section{Vector construction and generation of transgenic plants}

To create the ProBAG2:GUS construct, 1886 bp sequence upstream of the BAG2 gene start codon were PCR-amplified. The resulting fragment was inserted upstream of the GUS reporter gene in the pGreenlI0229-GUS vector via Xhol and Kpnl sites [19]. Similarly, ProBAG6:GUS was also created for this study, 1884 bp sequence upstream of the $B A G 6$ gene start codon were PCR-amplified. The resulting fragment was inserted upstream of the GUS reporter gene in the pGreenII0229-GUS vector via Xhol and $K p n l$ sites [19]. Primer sequences used in this experiment are available in Supplementary Table S1. These constructs were transformed into Agrobacterium tumefaciens strain C58C1 (pMP90/pJICSa-Rep). The Arabidopsis transgenic plants were generated using floral dip method [20].

\section{RT-PCR Analysis}

RT-PCR was performed to test the transcription levels of the $B A G 2$ and $B A G 6$ gene in mutant or wild-type (WT) plants. Total RNA was extracted from 7-day-old seedlings using Trizol reagent (TransGen Biotech, Beijing, China). The first-strand cDNA was synthesized using Easy Script First-Strand cDNA Synthesis Super Mix (TransGen Biotech, Beijing, China). The ACTIN2 gene was used as an internal control. The primer sequences used in this experiment are listed in Supplemental Table S1.

\section{GUS Histochemical Staining}

For GUS staining, various tissues of ProBAG2:GUS and ProBAG6:GUS transgenic plants were incubated for $12 \mathrm{~h}$ at $37^{\circ} \mathrm{C}$ in GUS staining buffer as previously described [21]. After staining, the tissues were 
cleared in chloral hydrate: distilled water: glycerol (8:3:1, w.v.v). Samples were visualized with microscopy (Olympus, bx63, Tokyo, Japan).

\section{Drought and ABA Treatments}

bag 2 and bag 6 single and bag 2 bag 6 double mutants and WT seeds were harvested at the same time and used for ABA treatments. In brief, surface-sterilized seeds were sown on MS medium plates supplemented without or with different concentrations of ABA (Sigma-Aldrich) as indicated. Plates were stratified at $4{ }^{\circ} \mathrm{C}$ in the dark for 72 hours and transferred to a growth incubator for normal conditions $\left(16 \mathrm{~h}\right.$ light $/ 8 \mathrm{~h}$ dark, $22^{\circ} \mathrm{C}$ ). For quantification of the percentage of seedlings with green cotyledons, seeds were sown on MS medium supplemented different concentrations of ABA and analyzed on the indicated days after stratification as previously described [22]. Pictures were scanned using HP LaserJet P1108 scanner.

For the drought tolerance test, 3-week-old plants grown in soil with sufficient water were treated with natural drought (water was withheld). After 14 days without watering, the drought-treated plants were rewatered and recovery was checked after 3 days. Drought experiments were repeated three times, and at least 9 plants for each individual genotype were used per experiment.

\section{Heat treatment and measurement of Chlorophyll content}

For heat treatment, 5-day-old WT, bag2, bag 6 and bag 2 bag 6 seedling were exposed to $45^{\circ} \mathrm{C}$ for 45 minutes. After three days recovery at $22^{\circ} \mathrm{C}$, seedlings survival rate was counted [1]. For the measurement of chlorophyll content, mesophyll cells were spectrophotometrically measured as previously reported [23].

\section{DAB and NBT Staining}

DAB and NBT staining were performed as previously described [24]. The experiment was performed three times and each time 20 seedlings of each genotype were stained. Five-day-old seedlings were used in this experiment.

\section{Hormone Treatment}

Hormonal treatment of the ProBAG2:GUS and ProBAG6:GUS seedling was performed as previously described [1].

\section{RT-qPCR}

For RT-qPCR analysis, of different stress related genes, we followed previously described protocol [1].

\section{Abbreviations}

ABA: Abscisic acid, ACC: 1-aminocyclopropane-1-carboxylicacid, JA: jasmonate, SA: salicylic acid, WT: wild type, GUS: $\beta$-glucuronidase, GA: Gibberellic acid

\section{Declarations}




\section{Ethics approval and consent to participate}

Not applicable.

\section{Consent for publication}

Not applicable.

\section{Availability of data and materials}

All data generated or analyzed during this study are included in this published article and its supplementary information files.

\section{Competing interests}

The authors declare that there is no conflict of interest.

\section{Funding}

This work was funded by the National Natural Science Foundation of China (Grants 31870230 , 31570247 , and 91417308 to SM). The funder had no role in the design of the study and collection, analysis, and interpretation of data and in writing the manuscript.

\section{Authors' contributions}

SM and YS conceived the project. SM planned and designed the research. MA performed experiments with the help of QL, ZL and LL. MA and SM analysed data and wrote the manuscript with contributions of all the authors. All authors have read and approved the manuscript.

\section{Acknowledgements}

We thank the Arabidopsis Biological Resource Center (ABRC) for providing mutant seeds, John Innes Centre for providing the pGREENIIO229 vector.

\section{References}

1. Nawkar GM, Maibam P, Park JH, Woo SG, Kim CY, Lee SY, et al. In silico study on ArabidopsisBAG gene expression in response to environmental stresses. Protoplasma. 2017;254:409-21. 
2. Kabbage M, Dickman MB. The BAG proteins: a ubiquitous family of chaperone regulators. Cell Mol Life Sci. 2008;65:1390-402.

3. Thanthrige N, Jain S, Bhowmik SD, Ferguson BJ, Kabbage M, Mundree S, et al. Centrality of BAGs in plant PCD, stress responses, and host defense. Trends Plant Sci. 2020;S1360-1385(20)30127-8.

4. Yan J, He C, Zhang H. The BAG-family proteins in Arabidopsis thaliana. Plant Sci. 2003;165:1-7.

5. Doukhanina EV, Chen S, van der Zalm E, Godzik A, Reed J, Dickman MB. Identification and functional characterization of the BAG protein family in Arabidopsis thaliana. J Biol Chem. 2006;281:18793801.

6. Fang S, Li L, Cui B, Men S, Shen Y, Yang X. Structural insight into plant programmed cell death mediated by BAG proteins in Arabidopsis thaliana. Acta Crystallogr D Biol Crystallogr. 2013;69:934-

7. Li L, Xing Y, Chang D, Fang S, Cui B, Li Q, et al. CaM/BAG5/Hsc70 signaling complex dynamically regulates leaf senescence. Sci Rep. 2016;6:1-2.

8. Lee DW, Kim SJ, Oh YJ, Choi B, Lee J, Hwang I. Arabidopsis BAG1 functions as a cofactor in Hsc70mediated proteasomal degradation of unimported plastid proteins. Mol Plant. 2016;9:1428-31.

9. You Q, Zhai K, Yang D, Yang W, Wu J, Liu J, et al. An E3 ubiquitin ligase-BAG protein module controls plant innate immunity and broad-spectrum disease resistance. Cell Host Microbe. 2016;20:758-69.

10. Locascio A, Marques MC, García-Martínez G, Corratgé-Faillie C, Andrés-Colás N, Rubio L, et al. BCL2ASSOCIATED ATHANOGENE4 regulates the KAT1 potassium channel and controls stomatal movement. Plant Physiol. 2019;181:1277-94.

11. Fu S, Li L, Kang H, Yang X, Men S, Shen Y. Chronic mitochondrial calcium elevation suppresses leaf senescence. Biochem Biophys Res Commun. 2017;487:672-7.

12. Echevarría-Zomeño S, Fernández-Calvino L, Castro-Sanz AB, López JA, Vázquez J, Castellano MM. Dissecting the proteome dynamics of the early heat stress response leading to plant survival or death in Arabidopsis. Plant Cell Environ. 2016;39:1264-78.

13. Fu C, Hou Y, Ge J, Zhang L, Liu X, Huo P, et al. Increased fes1a thermotolerance is induced by BAG6 knockout. Plant Mol Bio. 2019;100:73-82.

14. Kang CH, Jung WY, Kang YH, Kim JY, Kim DG, Jeong JC, et al., AtBAG6, a novel calmodulin-binding protein, induces programmed cell death in yeast and plants. Cell Death Differ. 2006;13:84-95.

15. Li Y, Kabbage M, Liu W, Dickman MB. Aspartyl protease-mediated cleavage of BAG6 is necessary for autophagy and fungal resistance in plants. Plant Cell. 2016;28:233-47.

16. Williams B, Kabbage M, Britt R, Dickman MB. AtBAG7, an Arabidopsis Bcl-2-associated athanogene, resides in the endoplasmic reticulum and is involved in the unfolded protein response. Proc Natl Acad Sci USA. 2010;107:6088-93.

17. Li Y, Williams B, Dickman M. Arabidopsis B-cell lymphoma2 (Bcl-2)-associated athanogene 7 (BAG7)-mediated heat tolerance requires translocation, sumoylation and binding to WRKY 29. New Phytol. 2017;214:695-705. 
18. Pan YJ, Liu L, Lin YC, Zu YG, Li LP, Tang ZH. Ethylene antagonizes salt-induced growth retardation and cell death process via transcriptional controlling of ethylene-, BAG- and senescence-associated genes in Arabidopsis. Front Plant Sci. 2016;7:696.

19. Bao S, Shen G, Li G, Liu Z, Arif M, Wei Q, et al. The Arabidopsis nucleoporin NUP1 is essential for megasporogenesis and early stages of pollen development. Plant Cell Rep. 2019;38:59-74.

20. Clough SJ, Bent AF. Floral dip: a simplified method for Agrobacterium-mediated transformation of Arabidopsis thaliana. Plant J. 1998;16:735-43.

21. Liu H, Liu B, Chen X, Zhu H, Zou C, Men S. AUX1 acts upstream of PIN2 in regulating root gravitropism. Biochem Biophys Res Commun. 2018;507:433-6.

22. Seo KI, Lee JH, Nezames CD, Zhong S, Song E, Byun MO, et al. ABD1 is an Arabidopsis DCAF substrate receptor for CUL4-DDB1-based E3 ligases that acts as a negative regulator of abscisic acid signaling. Plant Cell. 2014;26:695-711.

23. Arnon DI. Copper enzymes in isolated chloroplasts. Polyphenoloxidase in Beta vulgaris. Plant Physiol. 1949;24:1.

24. Yang L, Wang S, Sun L, Ruan M, Li S, He R, et al. Involvement of G6PD5 in ABA response during seed germination and root growth in Arabidopsis. BMC Plant Biol. 2019;19:1-4.

\section{Figures}



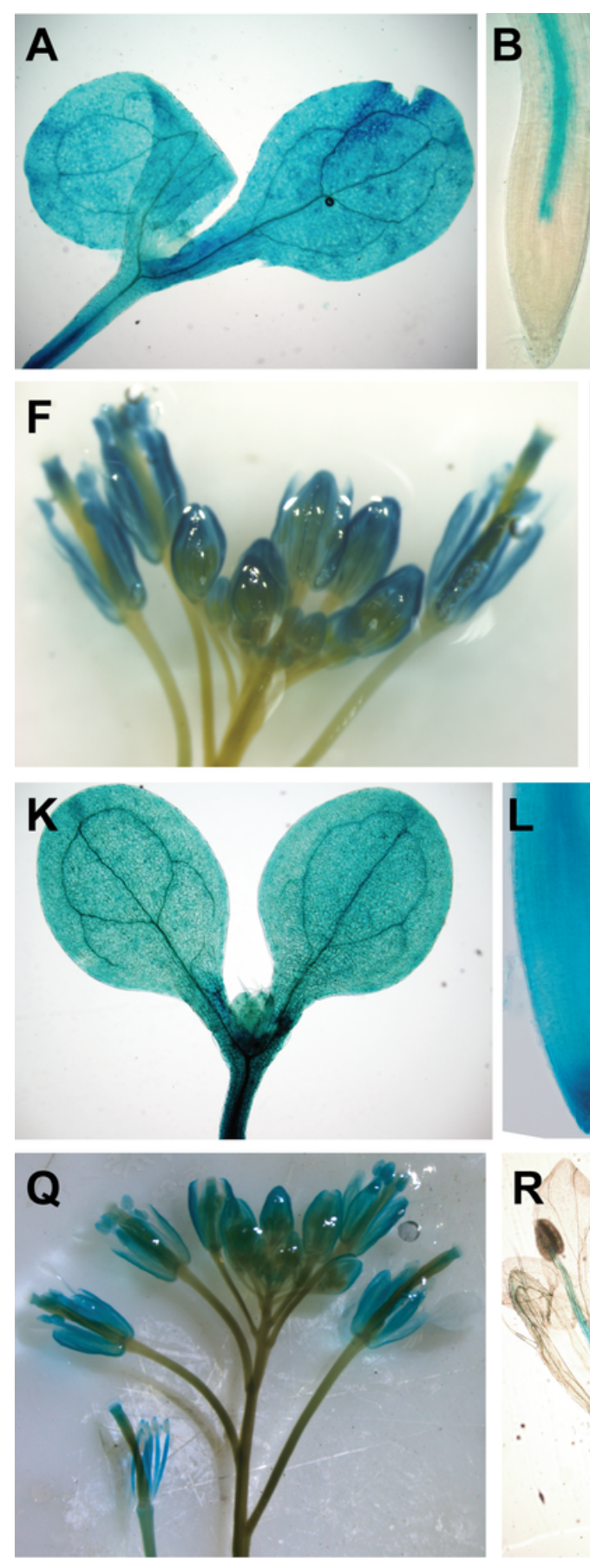
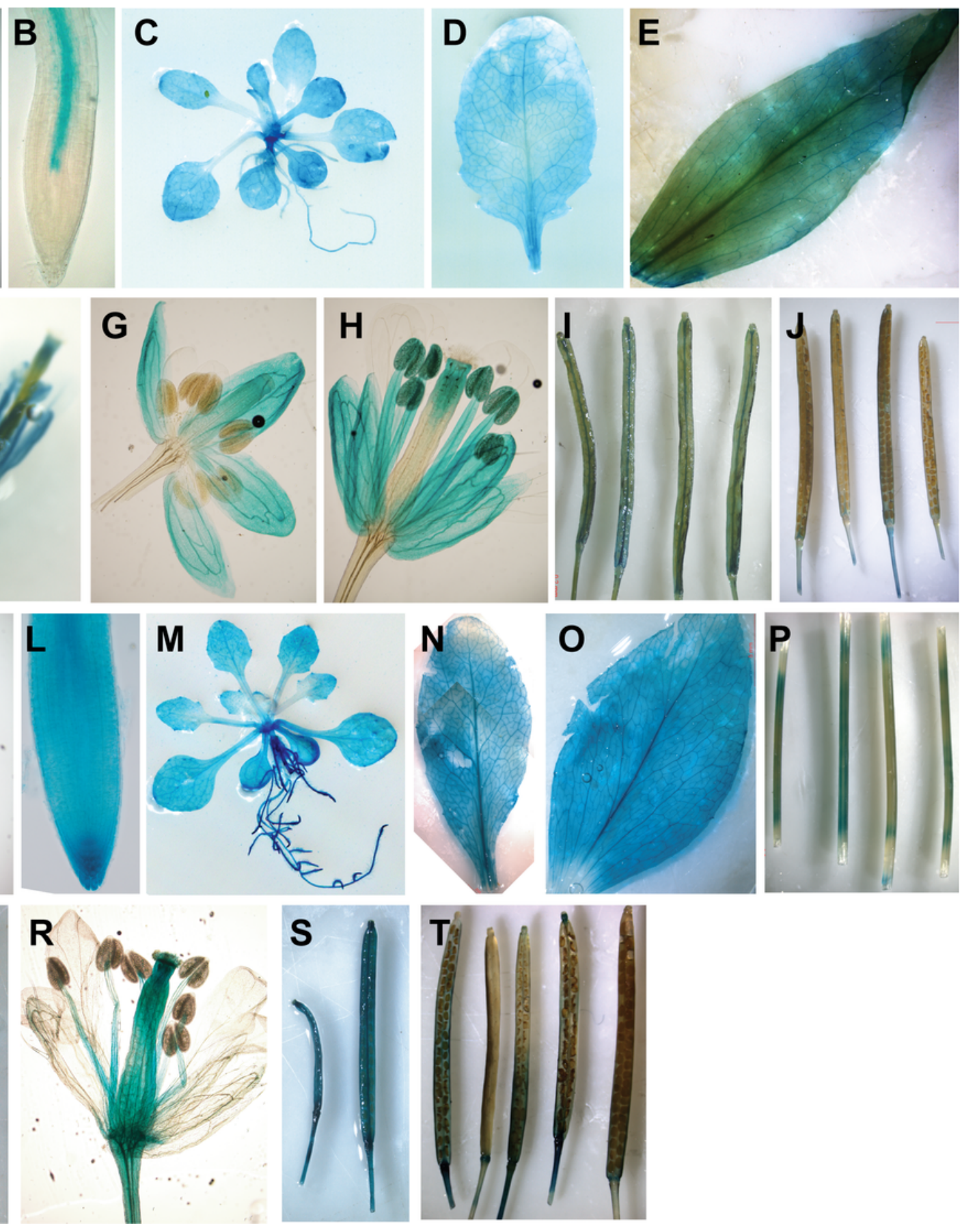

\section{Figure 1}

Expression patterns of the Arabidopsis BAG2 and BAG6 genes. a-j expression of PROBAG2:GUS in 5-dayold seedlings cotyledons and hypocotyl (a), root tip (b), 2-week-old plant (c), rosette leaf (d), cauline leaf (e), inflorescence (f), flower bud (g), opened flower (h), young siliques (i), and mature siliques (j). $k-t$ Expression of ProBAG6:GUS in cotyledons and hypocotyl ( $k$ ) and root tip (l) of 5-day-old seedling, in 2week-old plant $(m)$, rosette leaf $(n)$, cauline leaf $(0)$, stems $(p)$, inflorescence $(q)$, opened flower $(r)$, young siliques (s), and mature siliques (t). 
A
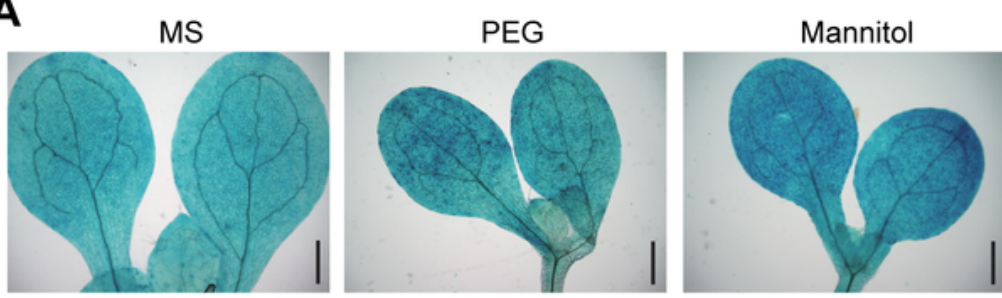

JA

ABA

SA
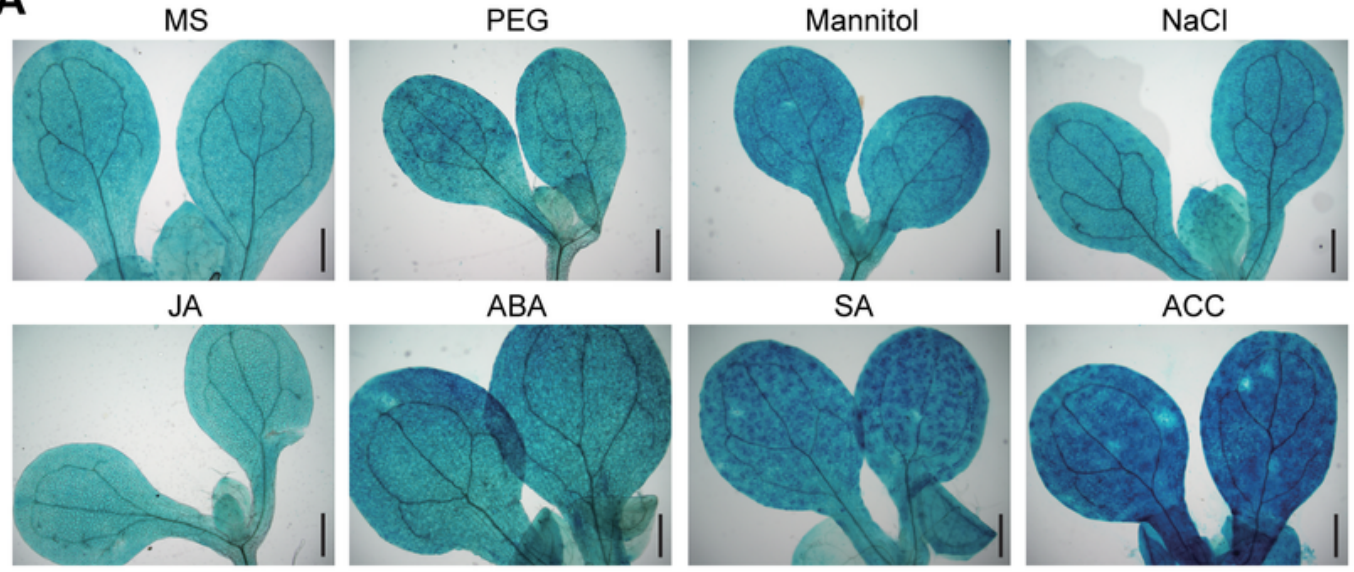

ACC

B

MS

PEG

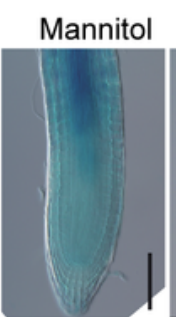

$\mathrm{NaCl}$

JA

ABA
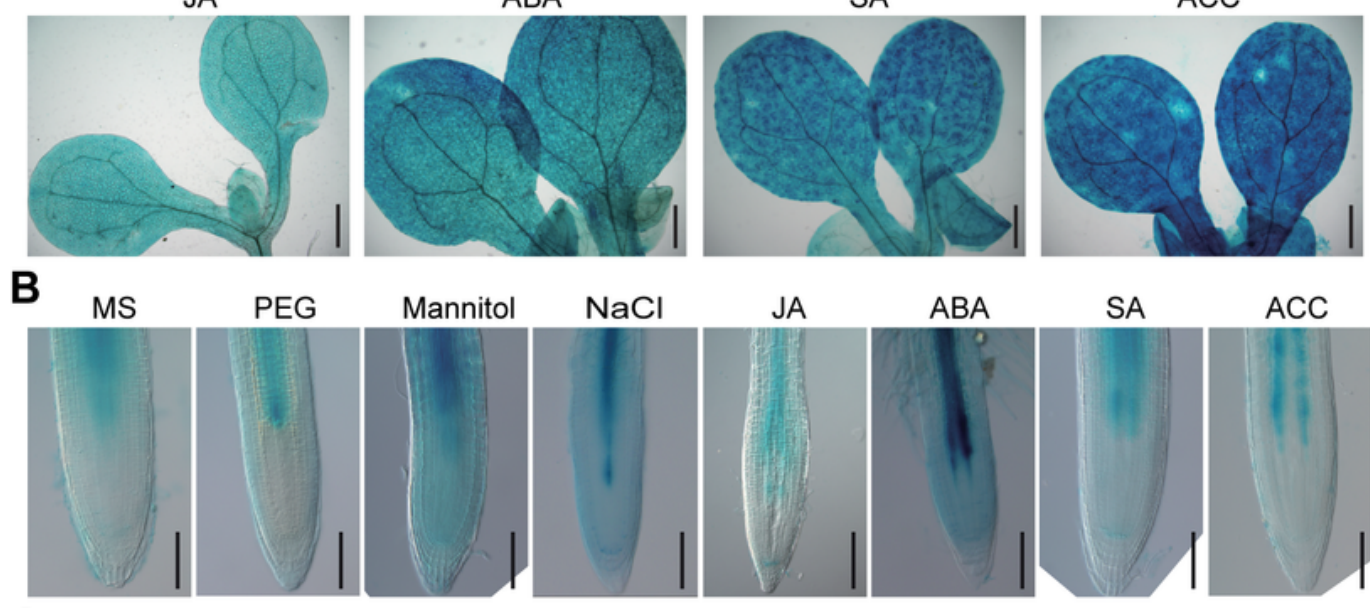

SA

ACC

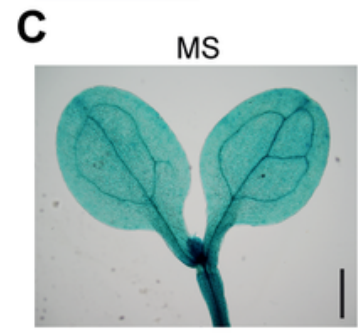

JA
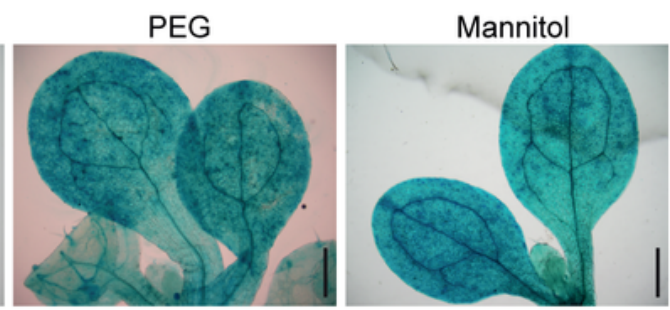

SA
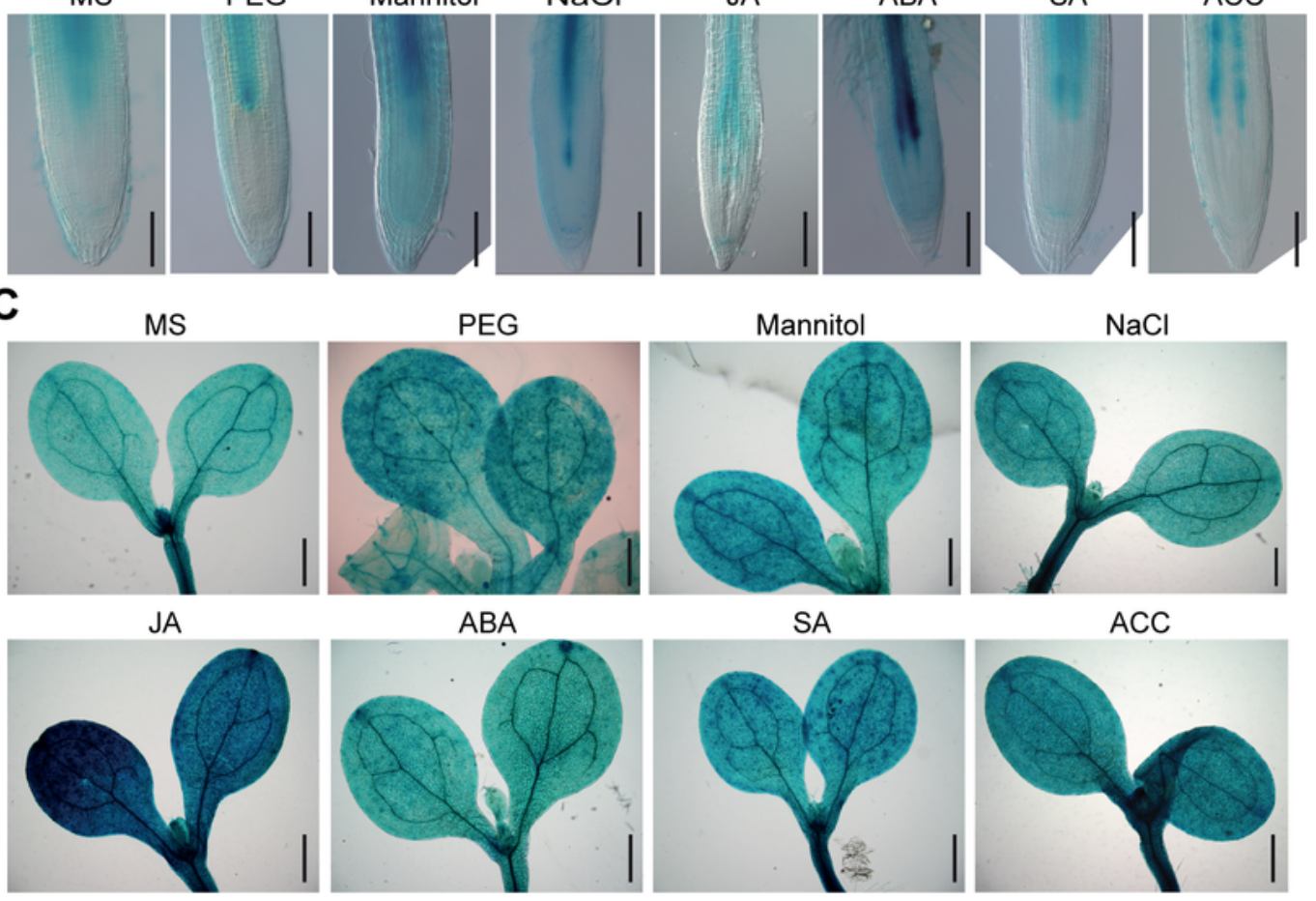

ABA
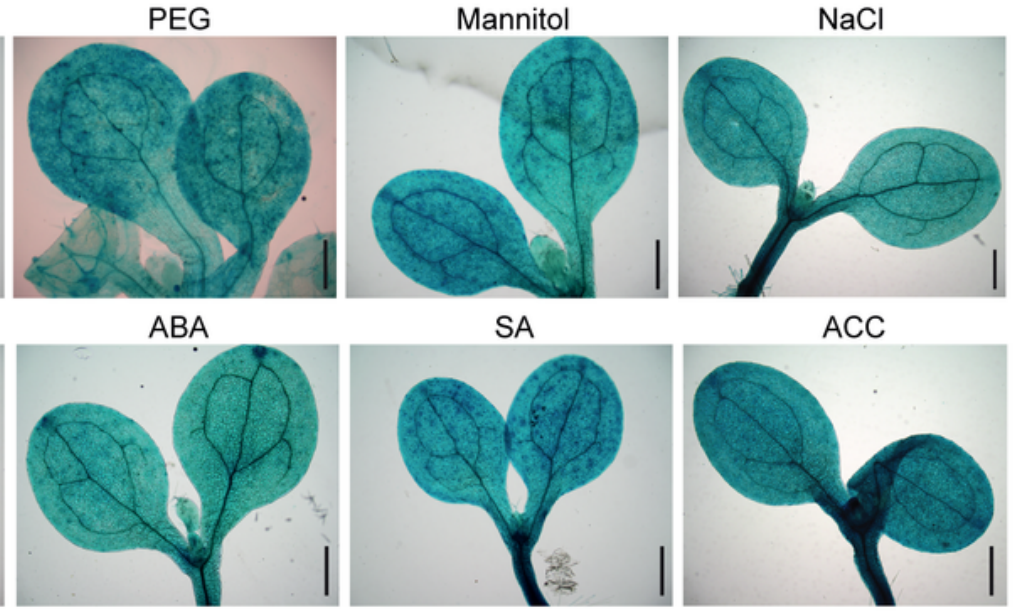

ACC
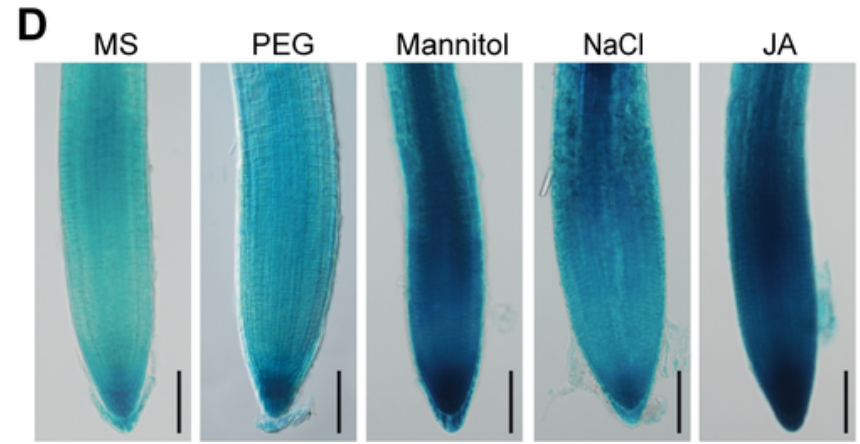

ABA
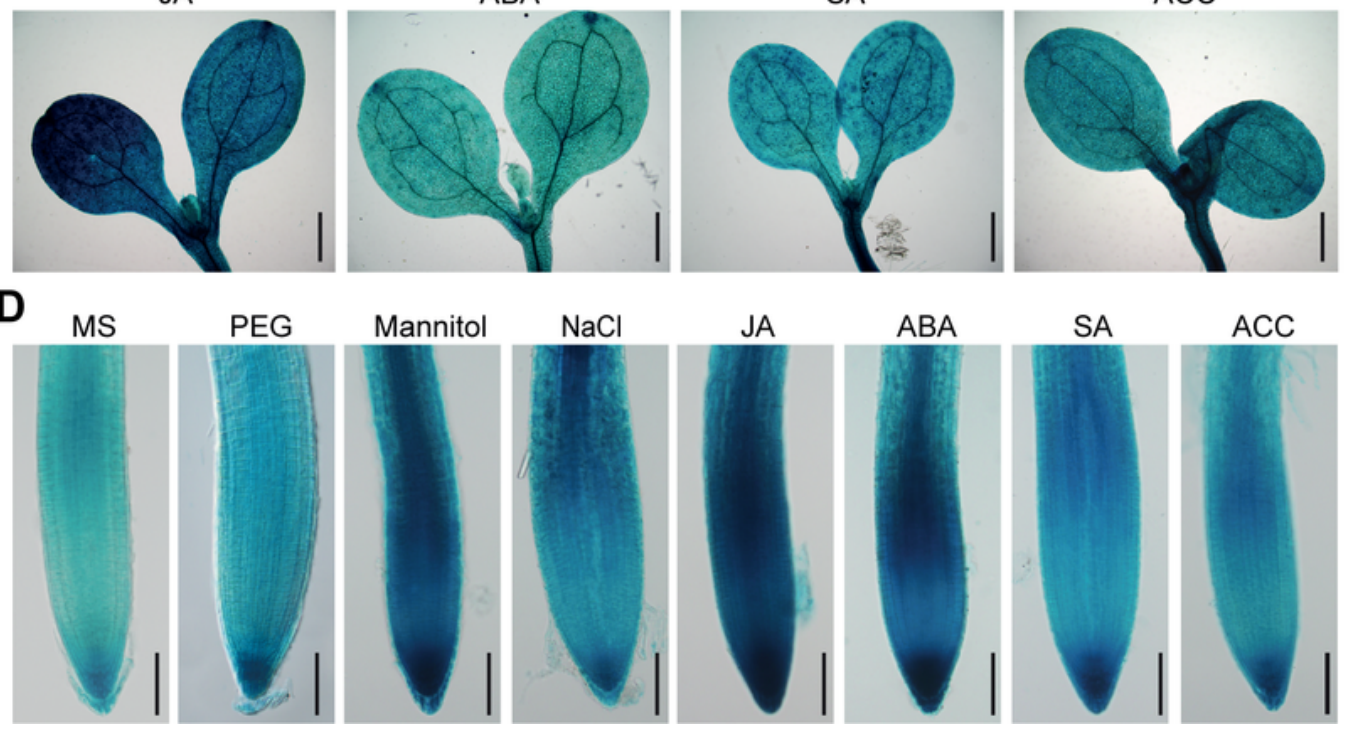

ACC

\section{Figure 2}

Expression of ProBAG2:GUS and ProBAG6:GUS in response to various abiotic stresses and hormones. Five-day-old ProBAG2:GUS ( $a$ and $b$ ) and ProBAG6:GUS ( $c$ and d) seedlings were transferred to MS media (mock) supplemented with 5\% PEG, $300 \mathrm{mM}$ mannitol, $200 \mathrm{mM} \mathrm{NaCl}, 10 \mu \mathrm{M} \mathrm{JA}, 10 \mu \mathrm{M} \mathrm{ABA}, 5 \mathrm{mM} \mathrm{SA}$ and $10 \mu \mathrm{M} \mathrm{ACC}$ and treated for $24 \mathrm{~h}$, then were collected for GUS staining. Shown are representative 
images of shoots ( $a$ and $c)$ and root tips ( $b$ and $d)$ of three independent experiments. Bars $=400 \mu \mathrm{m}$ in $(a$ and c) and $100 \mu \mathrm{m}$ in (b and d).

A

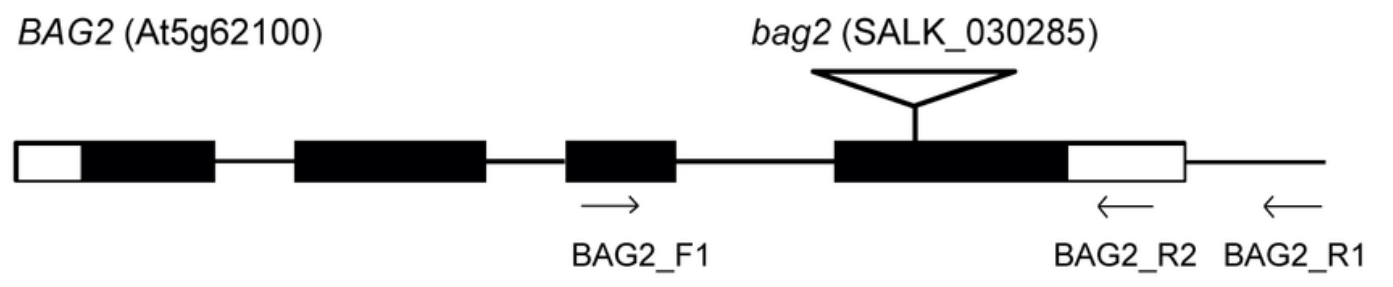

B

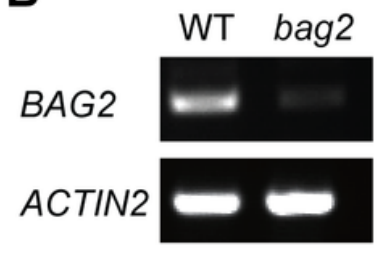

C

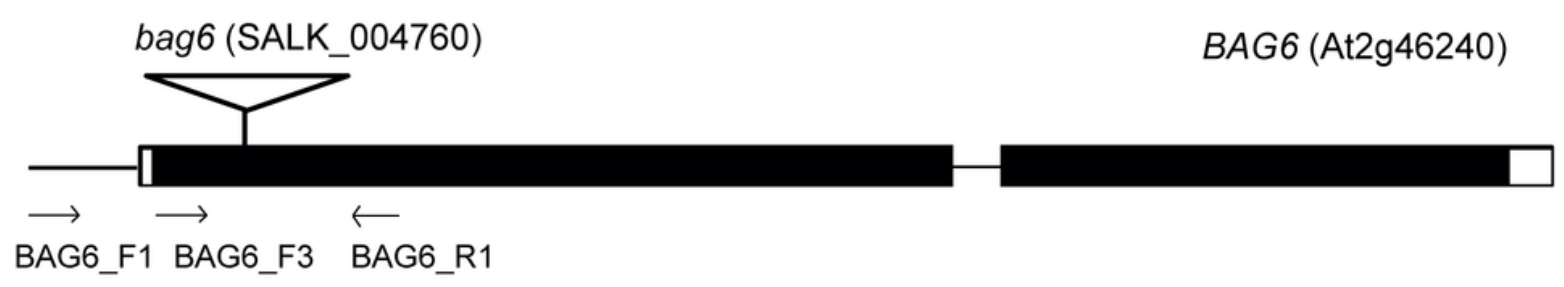

D

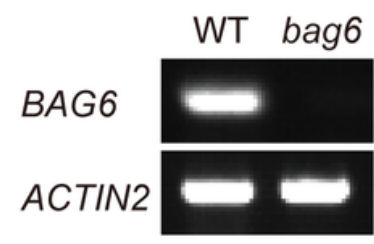

Figure 3

Insertion sites and transcription analysis of the bag2 and bag6 mutants. a and c Structures of the AtBAG2 (a) and the AtBAG6 (c) genes with T-DNA insertion sites. White boxes at left and right borders represent UTR regions, black boxes indicate exons, lines between black boxes represent introns, flags indicate the T-DNA insertion sites, and arrows indicate the positions of primers used for PCR verification of the insertions and for RT-PCR. b and d RT-PCR analysis of transcription levels of the AtBAG2 (b) and the AtBAG6 (d) genes in WT and bag2 or bag6 mutants. The ACTIN2 gene was used as the internal control. Total RNA was extracted from 7-day-old seedlings. The presented images in (b) and (d) were cropped and the original, full-length gel images were provided in the additional file Fig. S3. 


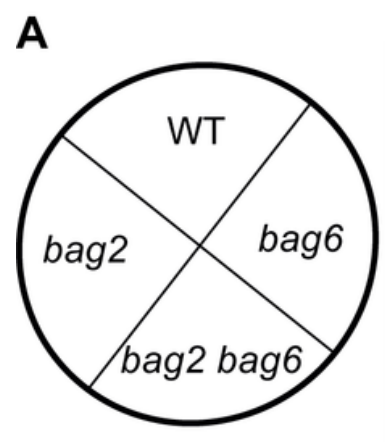

B

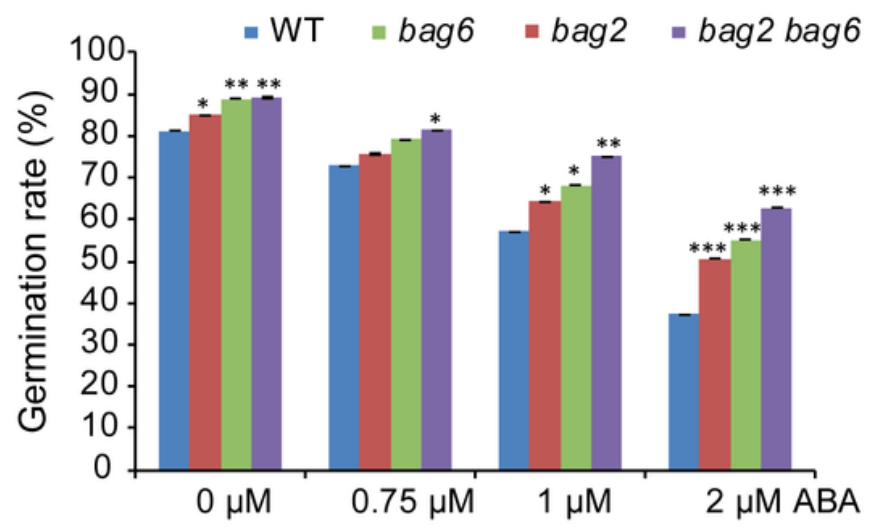

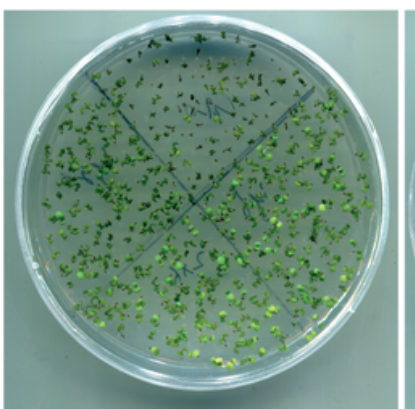

$0.75 \mu \mathrm{M}$

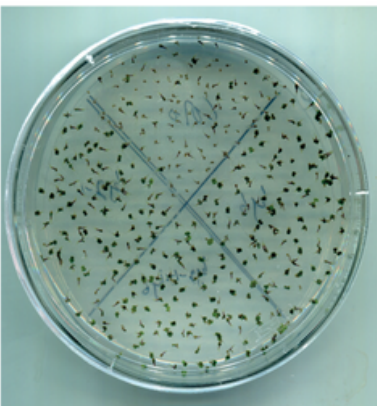

$1 \mu \mathrm{M}$

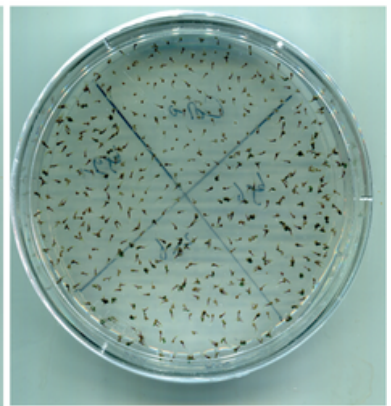

$2 \mu \mathrm{M} \quad \mathrm{ABA}$

C

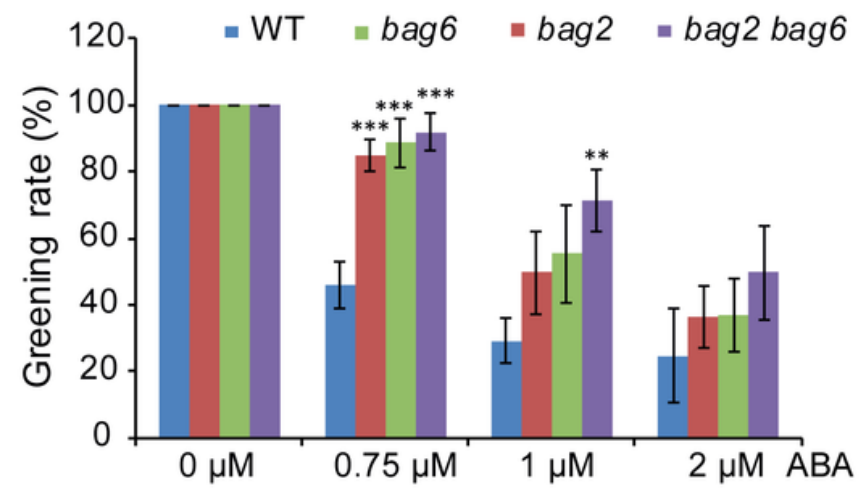

Figure 4

bag2 and bag6 mutants are less sensitive to ABA than the WT. a-c Seed germination rate and greening rate of WT, bag2 and bag6 single, and bag2 bag6 double mutants. Sterilized seeds were sowed on MS medium supplemented without or with different concentrations of ABA. The germination percentage and the greening rate were scored. All the data represent the means of three independent experiments \pm SD. *, represent the significant differences at $p<0.05$; ** represent $p<0.01$; ${ }^{\star \star \star}$, represent $p<0.001$ (Student's ttest). 
A
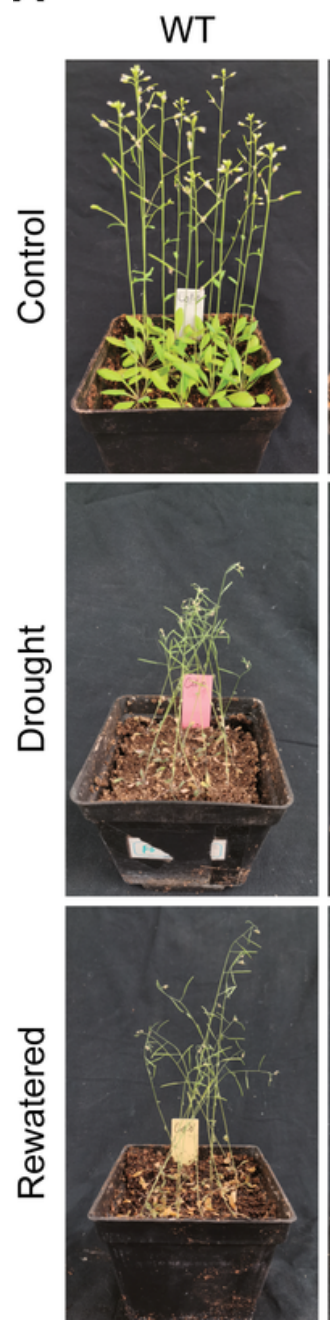
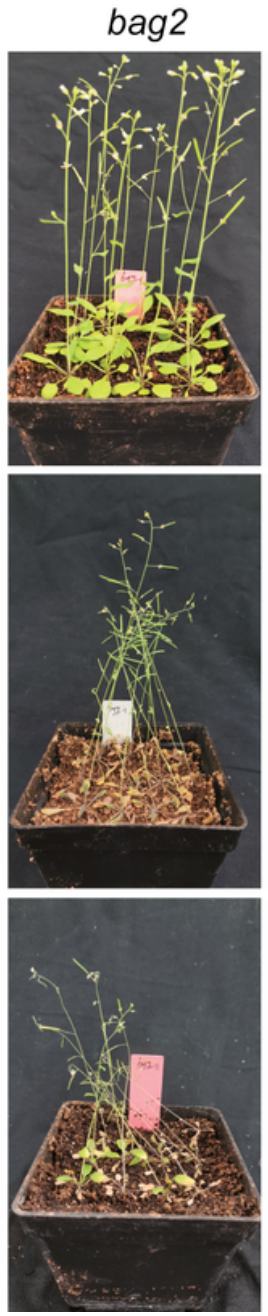
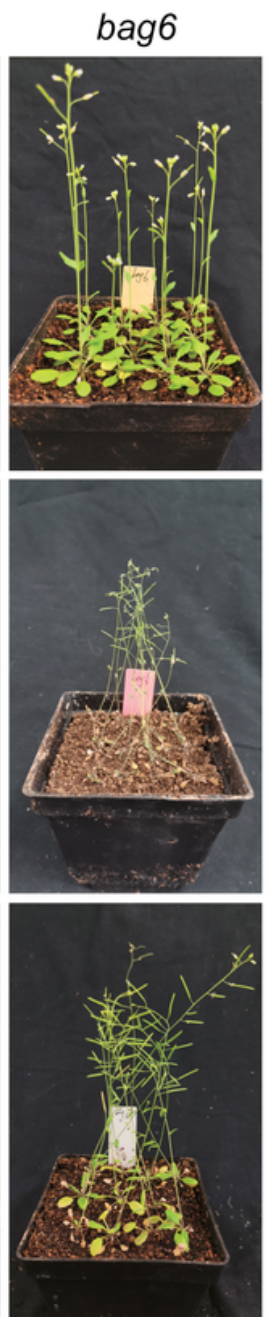
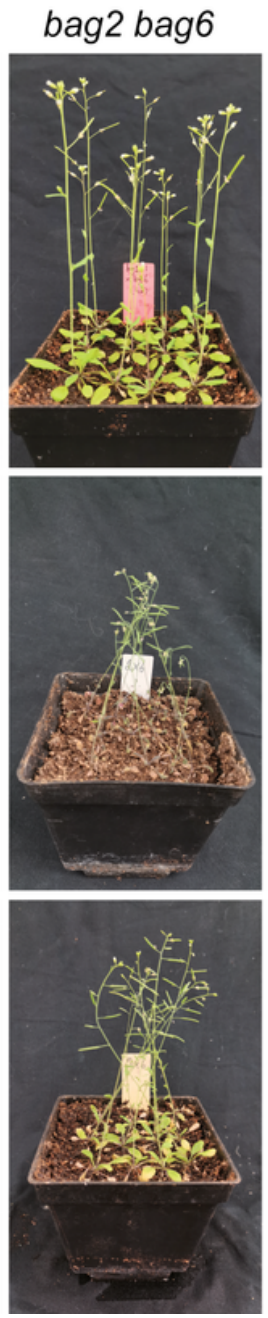

B
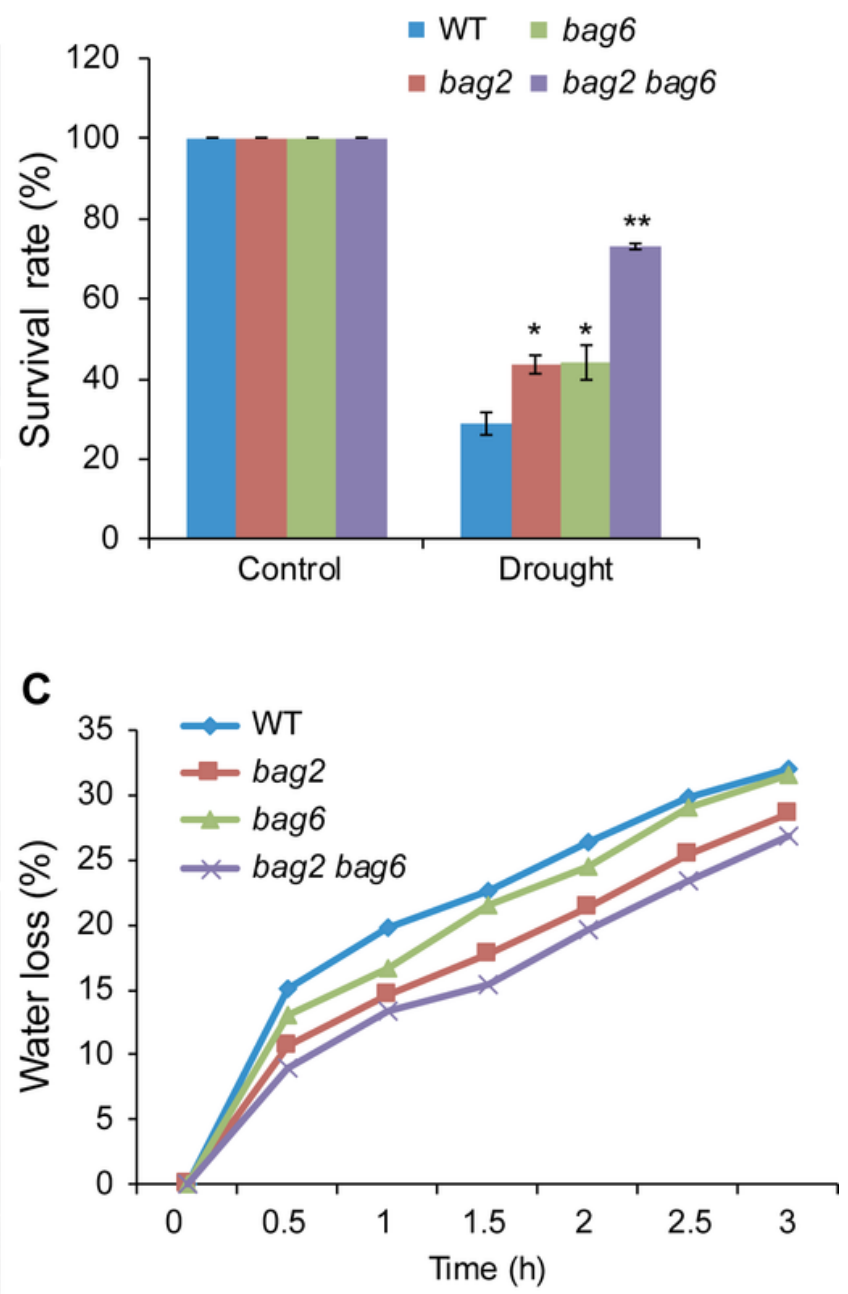

\section{Figure 5}

bag2 and bag6 mutants are more tolerant to drought. a Phenotypes of WT, bag2 and bag 6 single, and bag2 bag6 double mutant plants treated with drought stress. Three-week-old plants were subjected to drought stress for 14 days and re-watered for 3 days. b The survival rate of WT, bag2, bag6, and bag2 bag6 plants after 3 days of re-watering following the 14 days drought treatment. Values are means \pm SD ( $n=5$ independent experiments, 108 plants for each genotype in each independent experiment were used for analysis). c Water loss in WT, bag2, bag6, and bag2 bag6 plants. Leaves from 3-week-old plants were collected and subjected for water loss at different time interval. All the data represent the means \pm SD of five independent experiments. ${ }^{*}$, represent the significant differences at $p<0.05$; ${ }^{* *}$, represent $p<0.01$; $* \star *$, represent $p<0.001$ (Student's t-test). 


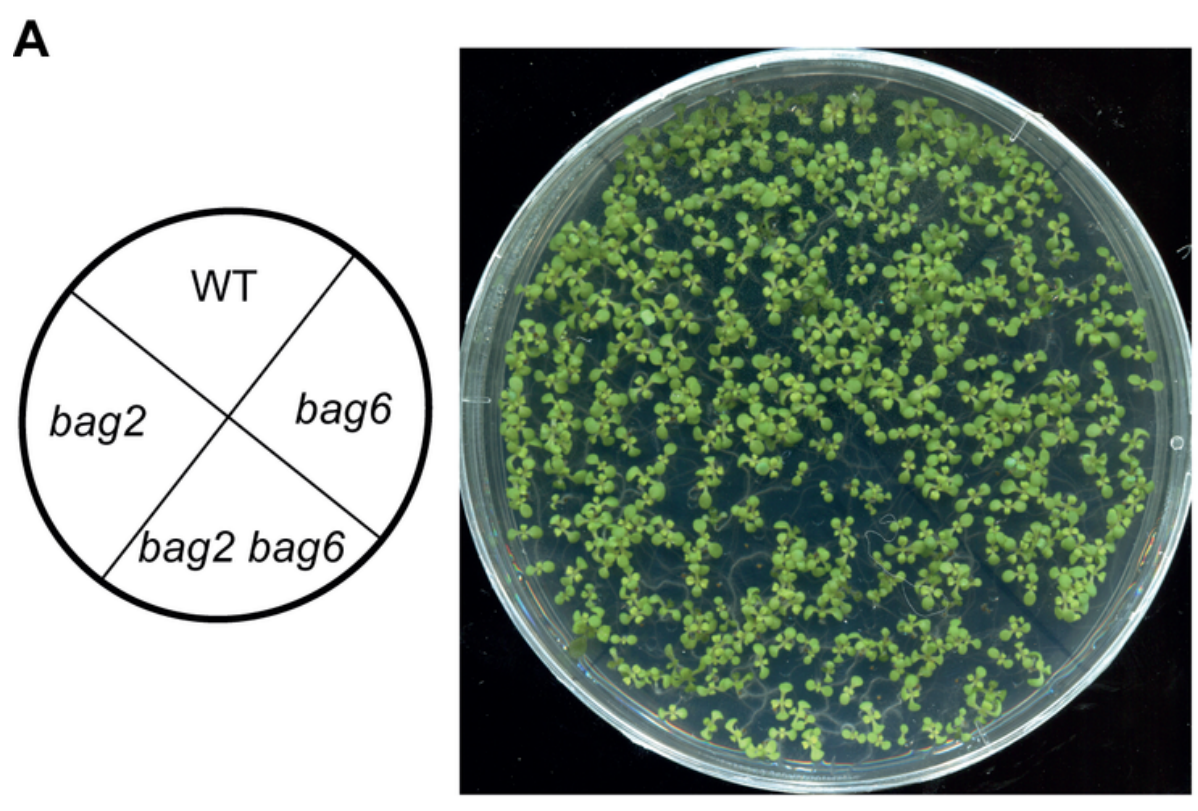

Untreated

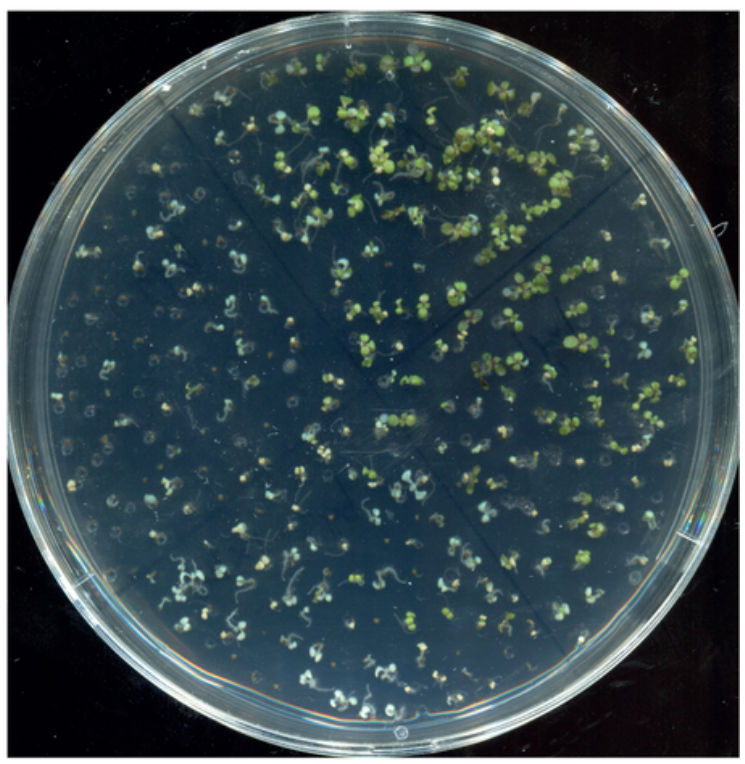

$45^{\circ} \mathrm{C}$ for $45 \mathrm{~min}$

B

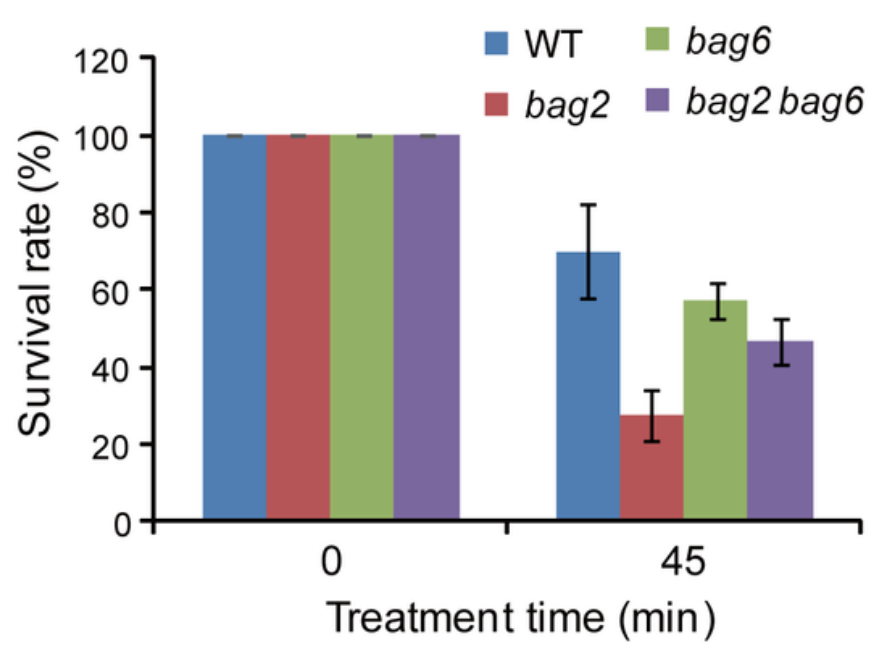

C

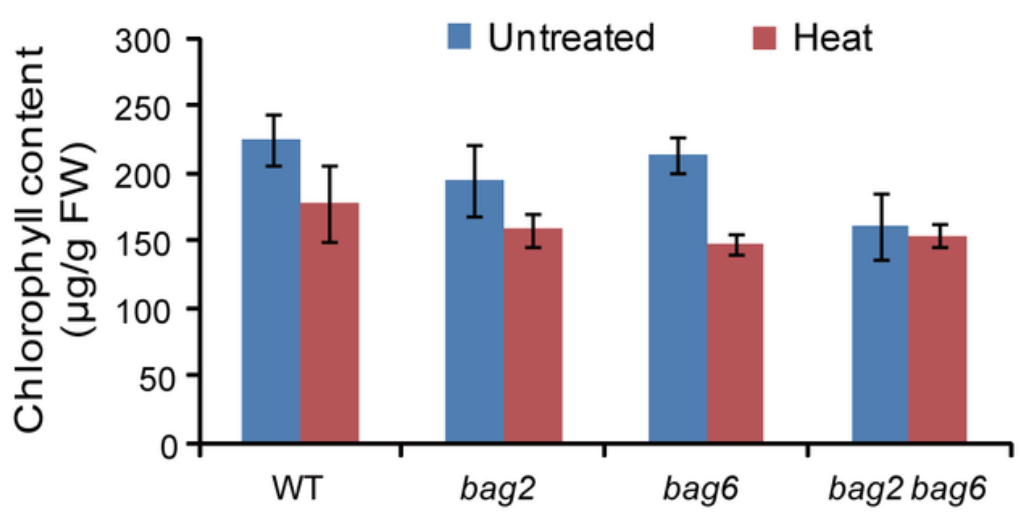

Figure 6

bag2 and bag6 mutants are sensitive to heat stress. a Phenotype of seedlings treated with heat stress. $b$ Survival rates of WT, bag2, bag6, and bag2 bag6 mutants under heat stress. Five-day-old seedlings were placed at $45{ }^{\circ} \mathrm{C}$ for 45 minutes and then resumed growth at $22{ }^{\circ} \mathrm{C}$. c Chlorophyll content measured from seedlings grown on normal growth conditions and after $45{ }^{\circ} \mathrm{C}$ heat stress. The data represent the means \pm SD of two independent experiments. 
A

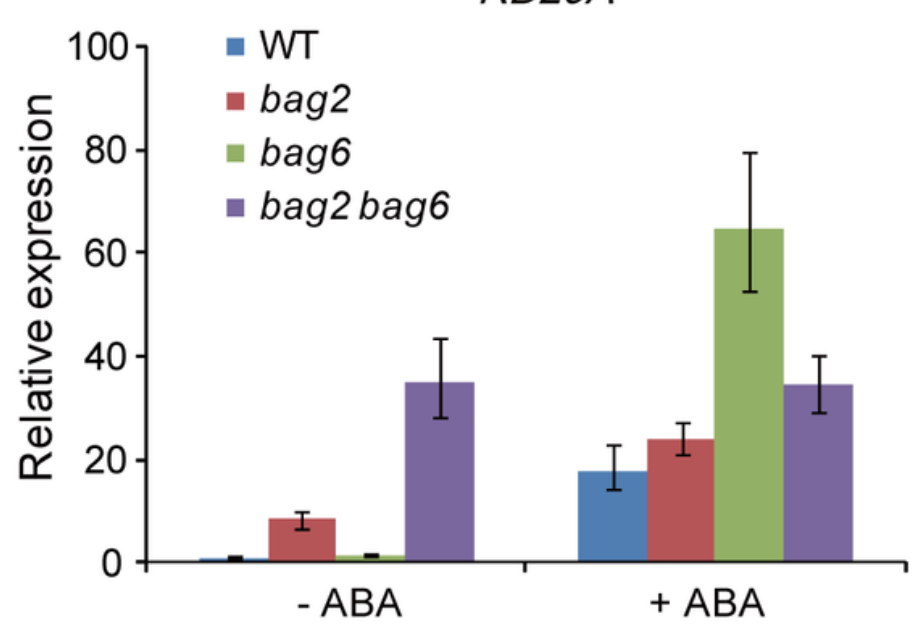

C

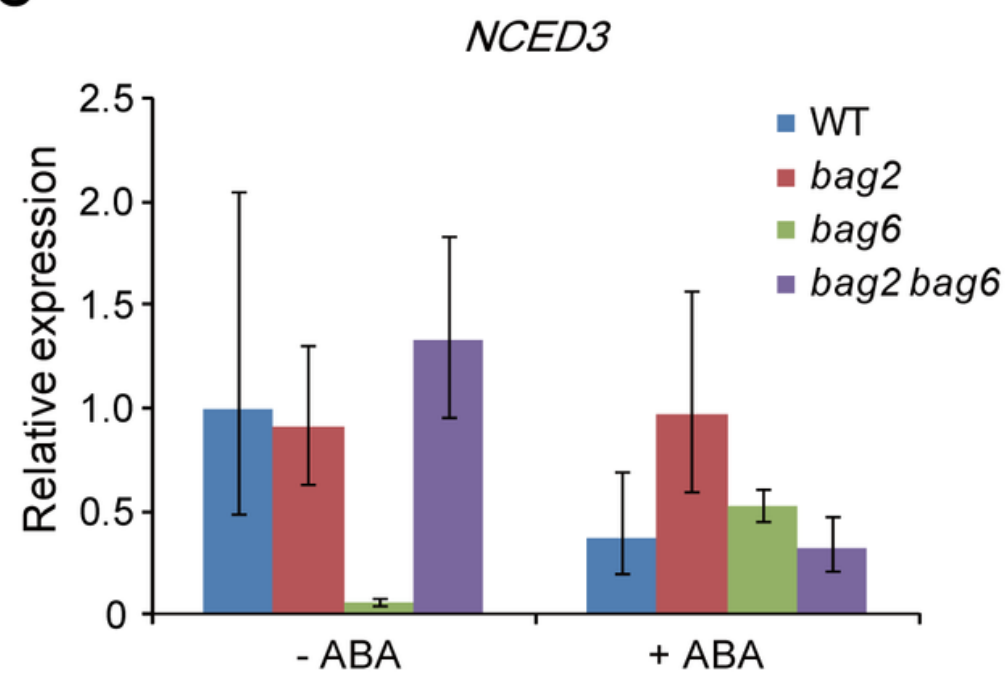

B

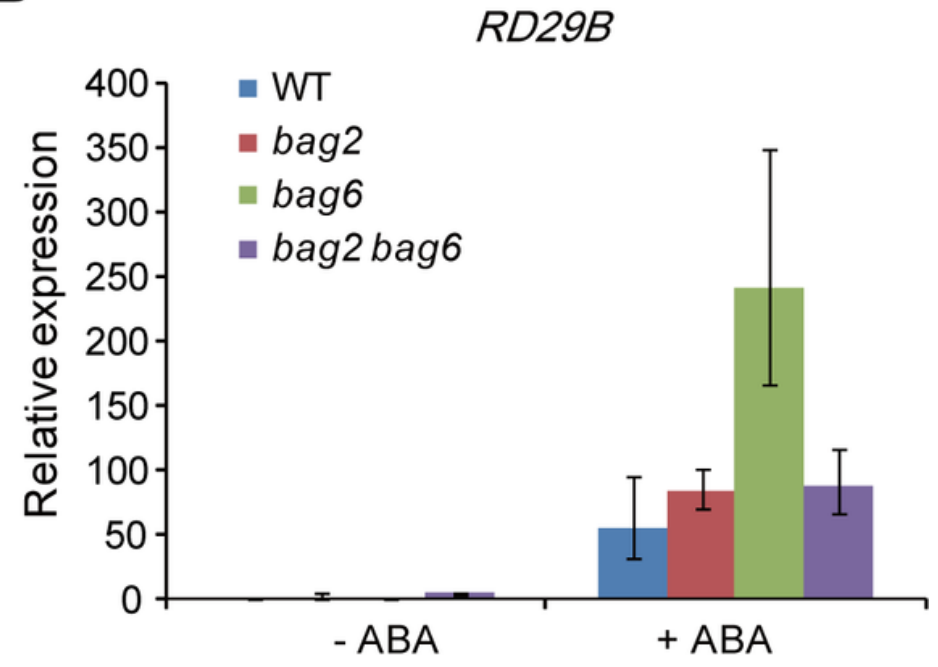

Figure 7

The relative expression of stress-related genes. a RD29A. b RD29B. c NCED3 were examined under control and $A B A$ conditions after 12 hours. Values are means \pm SD of three replicates. 
A
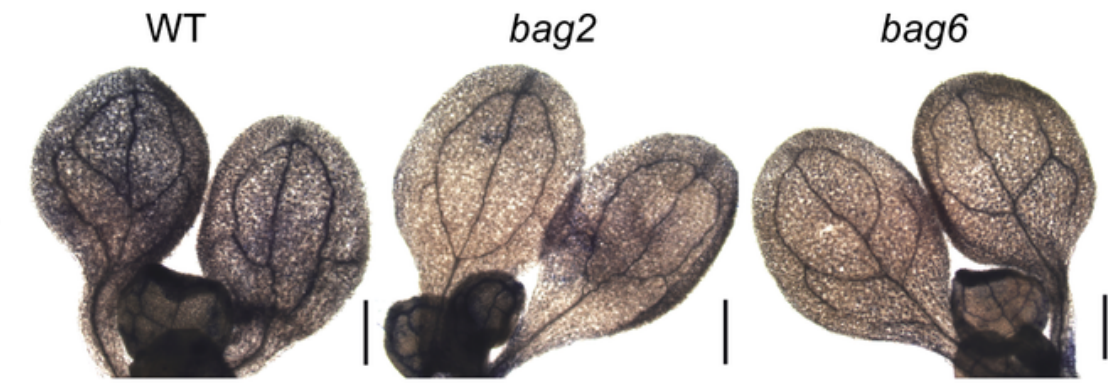

bag2 bag6

B
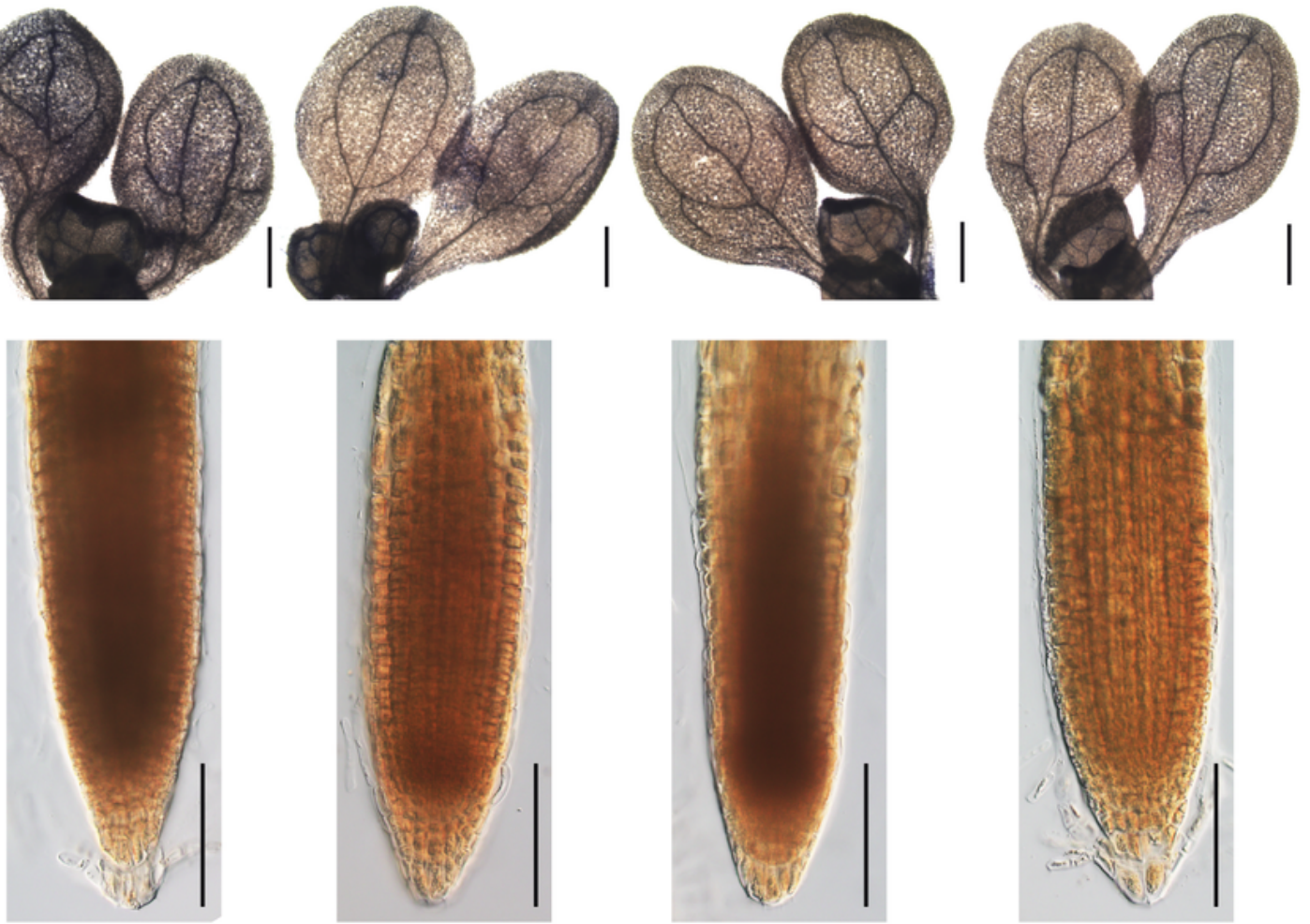

ABA

C
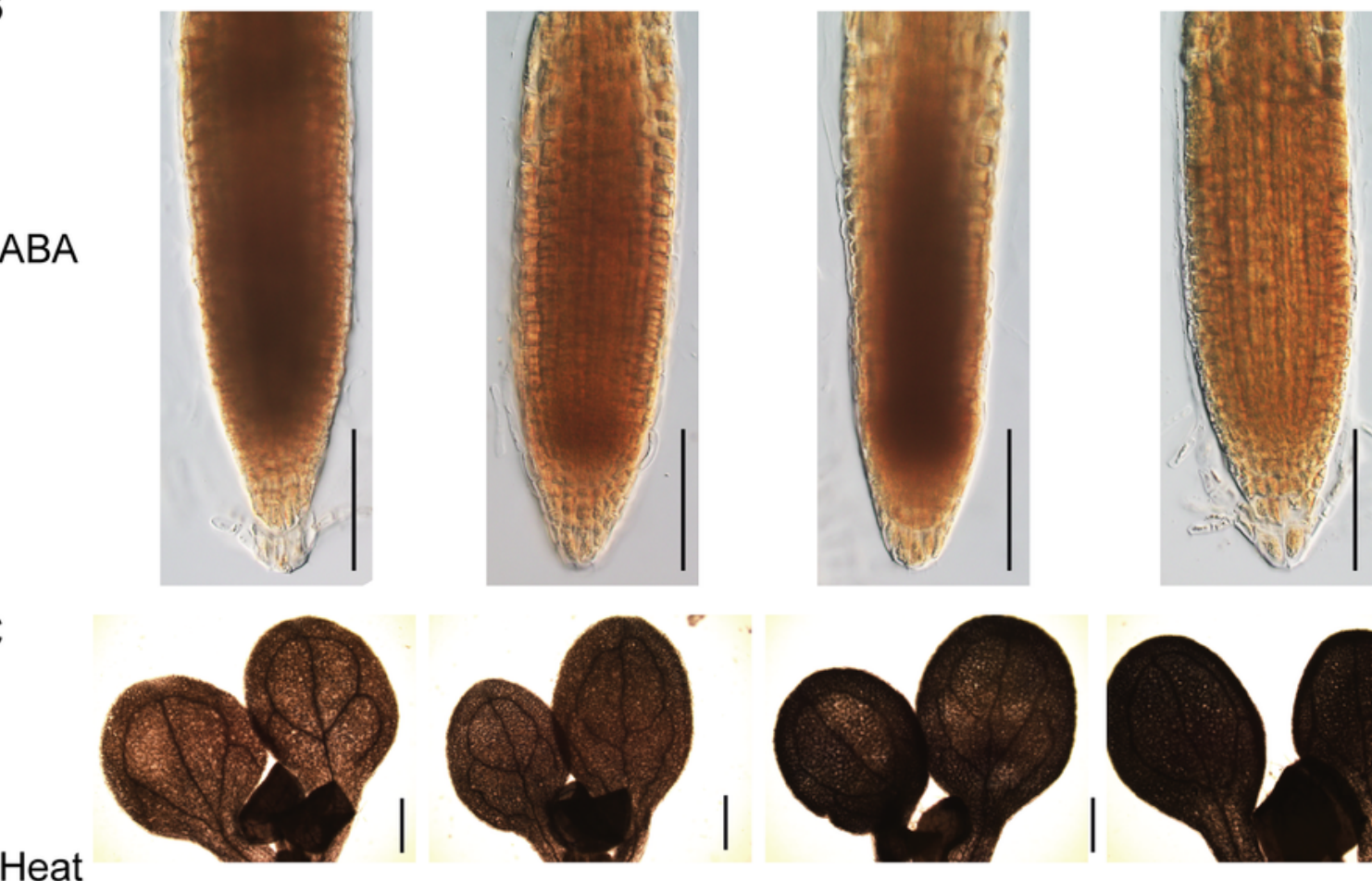

Heat
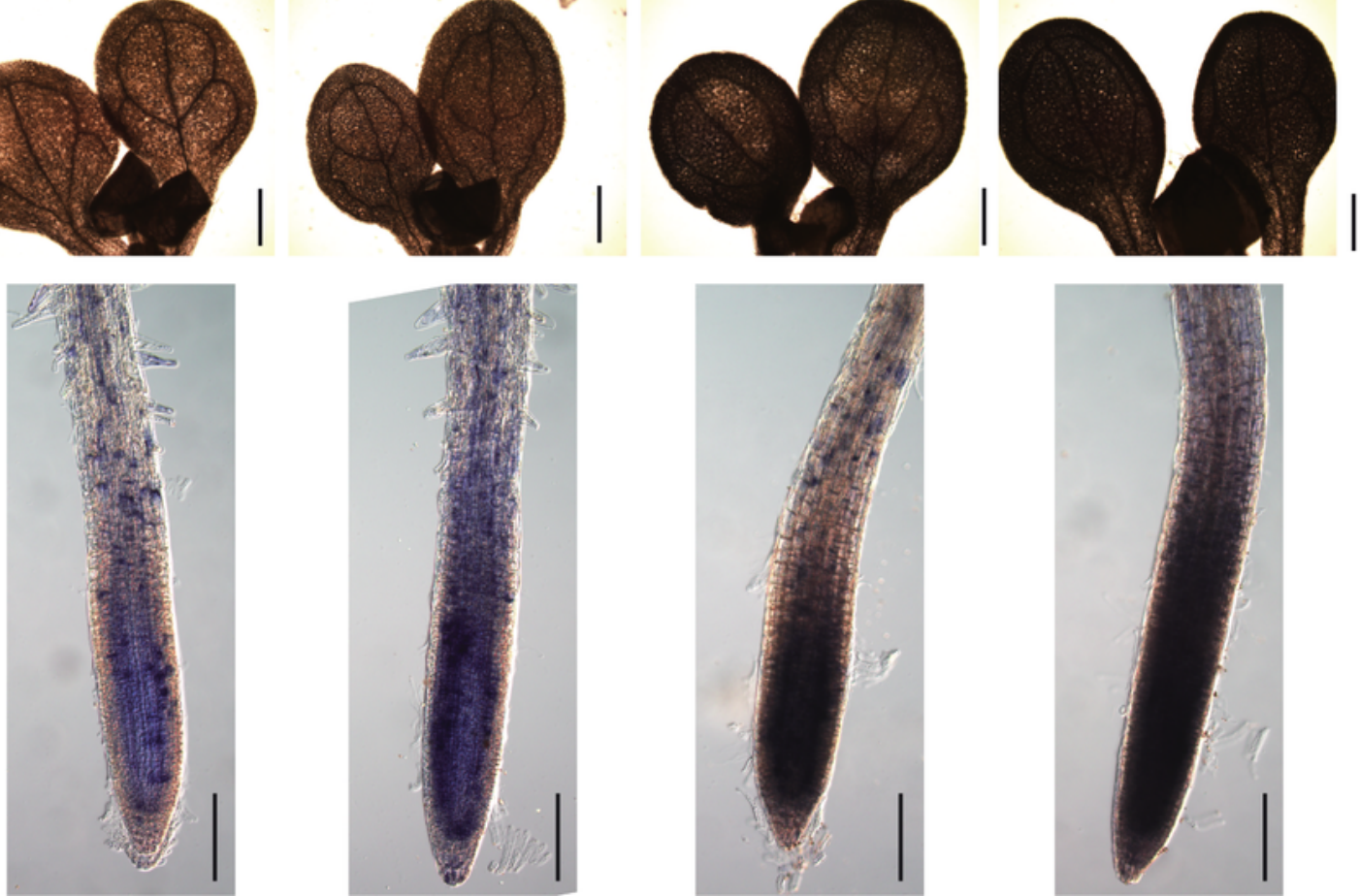

\section{Figure 8}

BAG2 and BAG6 mutation affects the ROS levels after ABA and heat treatment in Arabidopsis seedlings. Five-day-old seedlings were transferred to $1 / 2 \mathrm{MS}$ agar plates supplemented with $20 \mu \mathrm{M}$ ABA for $6 \mathrm{~h}$ or were treated with heat stress $\left(37^{\circ} \mathrm{C}\right)$ for $3 \mathrm{~h}$, then were stained with NBT (staining for 02.-) (a and c) or DAB (staining for $\mathrm{H} 2 \mathrm{O} 2$ ) (b). At least three independent experiments were performed for each analysis 
and 15 seedlings of each genotype were examined. Bars $=400 \mu \mathrm{m}$ in (a) and in the shoot images in (c) and $100 \mu \mathrm{m}$ in (b) and in the root images in (c).

\section{Supplementary Files}

This is a list of supplementary files associated with this preprint. Click to download.

- BAG26SupplementaryFig.sformatted.pdf 